Journal of Computer Science 8 (9): 1434-1440, 2012

ISSN 1549-3636

(C) 2012 Science Publications

\title{
Fuzzy Cost Enabled Cluster Based Multipath Routing Algorithm for Mobile Ad-Hoc Networks
}

\author{
${ }^{1}$ Gomathi, M. and ${ }^{2} \mathrm{G}$. Rajendran \\ ${ }^{1}$ Department of Computer Application, \\ ${ }^{2}$ Department of Mathematics, \\ Kongu Engineering College, Perundurai, Erode, 638052, India
}

\begin{abstract}
Problem statement: This article proposes the selection of best paths in Mobile Ad Hoc Network (MANET) based on multipath routing with fuzzy cost in respect of the Fuzzy Cost Enabled Cluster based Routing (FCECR). Approach: By implementing FCECR, we have made changes to reduce the cost and route discovery overhead for the selection of best path from multipath routing from source to destination node. Results: Results are found in NS2 simulator that cost factorinfluence the network traffic and bandwidth consumption. Besides, it exposes effective changes of network topology. Conclusion/Recommendations: It is shown that the proposed method is used to cluster hierarchal structure management to look for selection of effective path. Future work will be evaluated by implementing the algorithm related to other parallel architectures.
\end{abstract}

Key words: Fuzzy cost, Multipath, MANET, cluster, routing table, Fuzzy Cost Enabled Cluster Based Multipathath Routing (FCECR)

\section{INTRODUCTION}

In this Age of Globalization and Network System, movable ad hoc network takes much importance and involvement in communication satisfying the technological urge and need especially exchange merge. In this Age of Computer, mobile also takes an indispensable role. However, a MANET is nothing but an independent collection of mobile nodes linked by wireless unfixed infrastructure. In a MANET all mobile hosts cooperate with another host and share the resources to achieve goals. In the consideration of either security cost or quality, the wireless can be set in any available existing infrastructure which rarely meets application requirements. The network topology called MANET has fast changes owing to the high moment of nodes and it was much difficult to identify the routes which have message packets to use. As a matter of fact, this is kept under constrained bandwidth, limited power and difficulty. It throws challenges upon the architect of a routing strategy in wireless network. In this dynamic connectivity, to build and maintain multihop route adds complications in MANET.

In fact there are a large number of routing paths available from source to destination node. The path can be selected using any one of the algorithm such as Ad Hoc on-Demand Distance Vector (AODV), Dynamic
Source Routing (DSR), Destination Sequence Distance Vector (DSDV) and WRP(Wireless Routing Protocol). DSDV is a table driven routing scheme (Mohamed et al., 2008), is purely set for mobile ad hoc networks with small number of mobile nodes. DSDV guarantees loop free path. But DSDV can rarely suitable for highly dynamic network. It never controls traffic and region of the network without change in topology. AODV is helpful to handle unicast and multicast routing and it dynamically maintains loop-free routes (Hsi and Abdullah 2009). Simultaneously AODV creates a disadvantage that leads to unnecessary bandwidth consumption (Perkins and Bhagwat, 1994).

WRP protocol brings into light above mechanism which not only reduces route loop but also ensures reliable message exchange. Also it requires abundant memory storage and resources for the purpose of keeping its table as a result of high mobility the control overhead updates table entries. Since it suffers from limited scalability, the protocol does not appear to be suitable and ideal for the large mobile ad hoc networks.DSR makes use of source routing which depends upon the routing table at a frequency of each intermediate device. In respect of routing algorithm mentioned above, routes will fail. A new route is formed with evaluation from source to destination node. So, it would be better to distribute data packets along

Corresponding Author: Gomathi, M., Department of Computer Application, Research Scholar, Kongu Engineering College, Perundurai, Erode, 638052, India 
the existed distributed paths. Also these data transmit to involve in selecting effective and right route path. It should be monitored carefully and systematically so as to keep cost, time, traffic load and route discovery. It is clearly stated in the article that all the nodes in the network are categorized into master node and slave node. Themaster node has one hop keeping away from the other slave node. The master node keeps a table that takes the IP address of its neighbor master node into clean records. It is sure that master node gives out necessary information rather than check of each node.

The article focuses that concentration is best owed upon choosing suitable as well effective path based on the cluster based multipath routing with fuzzy cost, which is used to cluster hierarchal structure management to look for multiple paths. So it will be advisable and conducive to execute mobile ad hoc networks with proposed algorithm with a view to forming parallel architecture.

Fuzzy cost routing Algorithm:Each vector is assigned to calculate fuzzy cost as displayed in Table 1. One agrees with the technical view (Raju and Rmachandram, 2008) fuzzy cost based routing protocol takes the routing decision by considering the predefined fuzzy cost threshold value.

To utilize cluster algorithm and to construct hierarchical topology requires an ideal method because any problem can be at easy stroke, solved. When an adapted mobile cluster algorithm sustains the mobility and maintains stability.The clustering management activates tract deployment of network and dynamic reconstruction after topology transformation in order to extend support of the multihop and mobile feature of ad hoc network.

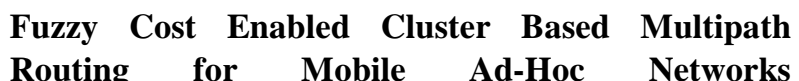
(FCECR):Fuzzy cost embedded with cluster based routing algorithm to form hierarchical topology may be a good method to find solutions for these problems. It bears the mobility and maintains the stability with robustness of network architecture. Fuzzy cost is to takes the routing decision through consideration of predefined threshold value and it can be defined if at all it depends on the requirement of path count. In support of the multihop and mobile features of mobile ad hoc network, the fast movement attached with deployment of network and proper reconstruction with topology change in an effective manner under clustering management.

Cluster Structure and Cluster Forming:In general all the nodes in the network are categorized into master node and slave node.

\begin{tabular}{|l|l|l|}
\hline Message type & length & Reserved word \\
\hline IP & \\
\hline IP (slave node) \\
\hline IP (neighbor master nodes) \\
\hline
\end{tabular}

Fig. 1: Master node

\begin{tabular}{|l|l|l|}
\hline Message type & length & Reserved word \\
\hline IP & & \\
\hline IP (master node) \\
\hline IP (master nodes can be heard) \\
\hline
\end{tabular}

Fig. 2: Slave node

Table 1: The Resources allocated to paths with total vector cost

\begin{tabular}{lllllll}
\hline $\begin{array}{l}\text { Vec } \\
\text { tor }\end{array}$ & $\begin{array}{l}\text { Wand } \\
\text { Width }\end{array}$ & $\begin{array}{l}\text { Comp } \\
\text { Effici } \\
\text { ency }\end{array}$ & $\begin{array}{l}\text { Power } \\
\text { Cons }\end{array}$ & $\begin{array}{l}\text { Traffic } \\
\text { load }\end{array}$ & $\begin{array}{l}\text { No of } \\
\text { Inter } \\
\text { Nodes }\end{array}$ & $\begin{array}{l}\text { Total } \\
\text { vector } \\
\text { cost }\end{array}$ \\
\hline 1 & 0.850 & 0.888 & 0.28 & 1.00 & 0.000 & 3.018 \\
2 & 0.571 & 0.777 & 0.42 & 0.25 & 0.500 & 2.518 \\
3 & 0.420 & 0.444 & 0.21 & 0.50 & 1.000 & 2.574 \\
4 & 0.280 & 1.000 & 0.00 & 0.75 & 0.770 & 2.800 \\
5 & 1.000 & 0.222 & 0.14 & 0.00 & 0.166 & 1.528 \\
\hline
\end{tabular}

In Fig. 1 and 2 every slave node belonging to one master node maintains the all sorts of IP address of its neighbor master node into record (Park and Yoo, 2010). When node exchange information using distributed push approach goes on, each and every node broadcasts a HELLO message regularly. Next, a slave node adds it IP address into its HELLO message. Similarly, a master node adds the IP address from the slave node.

In Fig. 3 and 4 to strengthen and to activate the master noderoute identity process with facilities, slave node keeps the IP address of the master node which can hear well. If master node moves away or a slave node fails to receive three HELLO packets continuously from its master node, it should be considered that the wireless link between them becomes broken. Thenslave node selects the new master node and records the IP address with fuzzy cost in accordance with that routing Table. As soon as a master node broadcasts the HELLO message to the right next packet, the selected master node gets information about the new slave node closely associated with its group. The slave node gets conformation of its new master node as it receives HELLO packet thatbears its IP address with fuzzy cost. In this table drawn below, the master node discovery process is neatly described. 


\begin{tabular}{|l|l|l|}
\hline Message type & length & Reserved word \\
\hline IP & \\
\hline IP (slave node) \\
\hline IP (neighbor master nodes) \\
\hline Fuzzy cost $\left(\mathrm{co}_{\mathrm{i}}\right)$ \\
\hline
\end{tabular}

Fig. 3: Master node with fuzzy cost

\begin{tabular}{|l|l|l|}
\hline Message type & length & Reserved word \\
\hline IP & \\
\hline IP (master node) \\
\hline IP (master nodes can be heard) \\
\hline Fuzzy cost $\left(\mathrm{co}_{\mathrm{i}}\right)$ \\
\hline
\end{tabular}

Fig. 4: Slave nodewith fuzzy cost

Proposed algorithm:Evaluation of Fuzzy Cost will be made through application of fuzzy measure function $\mu$ to the resources. It shows the resource values into 0 to 1 interval having reliable or unreliable resource for routing, corresponding cost vector is $\mathrm{co}_{\mathrm{i}}$ and $\mathrm{M}_{\mathrm{i}}$ is resource vector will be calculated by applying fuzzy measure $\mu$.

Let So be an source node, Dn be an destination nodeand $\mathrm{M}_{\mathrm{i}}$ is resource vector $\left(\lambda 1_{\mathrm{i}}, \lambda 2_{\mathrm{i}}, \lambda 3_{\mathrm{i}}, \ldots \ldots . . . \mathrm{k}_{\mathrm{i}}\right) \mathrm{k}$ number of resources are regarded $k$. some of them may be reliable and unreliable for routing. A few of them available band width is reliable for routing and traffic is unreliable for routing. In this method using several metrics has been selected to meet these salient principles and to produce a single cost metric $\left(\mathrm{co}_{\mathrm{i}}\right)$ for selecting paths. The various routing metrics used are: bandwidth, computing efficiency, power consumption, traffic load and the number of hops and cost vector.

In a computer network shown in a graph $\mathrm{G}=(\mathrm{N}, \mathrm{E})$, where $\mathrm{n}$ is set of nodes and $\mathrm{E}$ is set of edges (links).Let So be the source node while Dn is the destination node. A cluster is marked by $\mathrm{CL}_{\mathrm{i}}=\{\mathrm{V}$. $\}$, where $\mathrm{SN}$ is the member of cluster i. Let $\mathrm{MN}_{\mathrm{i}}$ be the master node $\mathrm{ij}^{\mathrm{ij}} \mathrm{CL}$. CBMRP is put into definition as the successor set of node $\mathrm{SN}_{\text {in cluster }} \mathrm{CL}_{\mathrm{i}}$ as $\mathrm{So}_{\mathrm{ij}}$ and the predecessor set as $\mathrm{Dn}_{\mathrm{ij}}$. When a source node $\mathrm{So}$ (So $€ \mathrm{CL}_{1}$ ) seeks to set up a link to a destination Dn, Source node (So) passes a route request message (RREQUEST) to its master nodeMN. If $\mathrm{Dn}$ is a member of cluster $\mathrm{CL}_{1}$ and hears the request message, then
Step 1: It can be set up multiple paths from source node Soto next hop nodes Dn $\left(\mathrm{SN}_{\mathrm{ij}}\right)=\left\{\mathrm{CL}_{\mathrm{i}^{-}}\right.$ $\left.\mathrm{SN}_{\mathrm{ij}}, \mathrm{i}=1, \mathrm{SN}_{\mathrm{ij}}=\mathrm{So}\right\}$.

Step 2: Then may set up multiple path from the source nodes So $\left(\mathrm{SL}_{\mathrm{ij}}=\left\{\mathrm{CL}_{\mathrm{i}}-\mathrm{SL}_{\mathrm{ij}}, \mathrm{i}=1, \mathrm{SL}_{\mathrm{ij}}=\mathrm{Dn}\right\}\right.$ todestination node Dn.

Step 3: $\mathrm{P}(\mathrm{Path})$ is reliable. The value of $\mu \mathrm{A}(\mathrm{z})=1$. That means $\mathrm{z}$ is completly member of fuzzy set. Then cost can be calculated upon the following formula.

$$
\left.\mathrm{CL}_{\mathrm{i}}^{\mathrm{p}}=\mathrm{Ni}(\mathrm{Mi}(\lambda \mathrm{p})) / \max \left\{\mathrm{N}_{\mathrm{i}}(\lambda \mathrm{p})\right\}_{\mathrm{i}=1}^{\mathrm{n}}\right)
$$

The loop will continue until $\mathrm{P}$ value reaches $\mathrm{L}$ and $\mathrm{i}$ value is reachesV.

Step 4: $\mathrm{P}$ is unreliable. The value of $\mu \mathrm{A}(\mathrm{z})=0 . \mathrm{z}$ whichmeant is noncompletly member of fuzzyset. Then cost can be calculated upon thefollowing formula:

$$
\left.\mathrm{CL}_{\mathrm{i}}^{\mathrm{p}}=\mathrm{Ni}(\mathrm{Mi}(\lambda \mathrm{p})) / \max \left\{\mathrm{N}_{\mathrm{i}}(\lambda \mathrm{p})\right\}_{\mathrm{i}=1}^{\mathrm{n}}\right)
$$

The loop will continue until $\mathrm{P}$ value reachesL and $\mathrm{i}$ value is reaches $C$.

Step 5: $i$ value ranges 1 to $C$ and $P$ value ranges from 1 to $\mathrm{L}$ (Maximum resources). The total cost can be calculated on the basics of following formula:

$$
\begin{aligned}
& \cos \mathrm{t}=0 ; \\
& \cos \mathrm{t}=\mathrm{CL}_{\mathrm{i}}^{\mathrm{p}}+\cos \mathrm{t} ; \\
& \cos \mathrm{t}[\mathrm{i}] \quad=\mathrm{cos} t
\end{aligned}
$$

Repeat the loop until reaches the maximum value i.e., $\mathrm{P}$ reaches $\mathrm{L}$ value and ireaches $\mathrm{C}$ value.

Step 6: It selects all the reliable link disjoint paths from So to So to $\operatorname{Dn}\left(\mathrm{co} \geq \mathrm{co}_{\text {lower }}\right)$, where,co isfavourable and $\mathrm{Co}_{\text {lower }}$ is member with low gradewith co(fuzzy cost).

Step 7: If all paths have been established, then it selects all the maximam-disjoint and loop- free reliable paths that satisfy above conditions.

If destination node Dn is not in the same cluster as source node So.

Step 1: source node So sends a route request message (RREQUST) to its master node $\mathrm{MN}_{1} \cdot \mathrm{MN}_{1}$ looks for which cluster the destination node Dn belongs to, the searches for a stable route as a directional guideline $\{$ So, $\left.\mathrm{CL}_{2}, \ldots, \mathrm{CL}_{\mathrm{m}-1}, \mathrm{Dn}\right\}$. At the same time, it sets up multiple links from source node So to the destination nodes set $\operatorname{Dn}\left(\mathrm{SN}_{\mathrm{ij}=}=\left\{\mathrm{CL}_{\mathrm{i}^{-}}\right.\right.$So, i=1\}, nodes set $\operatorname{Dn}\left(\mathrm{SN}_{\mathrm{ij}}\right)=\left\{\mathrm{MN}_{\mathrm{i}^{-}} \quad \Sigma \mathrm{SN}_{\mathrm{ij}}, \Sigma \mathrm{SN}_{\mathrm{ij}}\right.$ denoted as nodes set between source node $\mathrm{So}$ and $\left.\mathrm{SN}_{\mathrm{ij}}, \mathrm{i}=1\right\}$ as next hop address, the hop of the links is likely more than one. 
Step 2: Master nodeMN ${ }_{1}$ sends the RREQUEST message to its next cluster $\mathrm{MN}_{2}$. Once $\mathrm{MN}_{2}$ receives this message, it will send the RREQUEST to next cluster and report the IP addresses of its slave nodes to $\mathrm{MN}_{1}$ at one time.

Step 3: Then, it sets up disjoint links: $\left\{\mathrm{N}_{1} \rightarrow \mathrm{N}_{2}\right\}:\left(\mathrm{N}_{1} €\right.$ $\mathrm{MN}_{1}, \mathrm{~N}_{2} € \mathrm{MN}_{2}$ );

Step 4: $\mathrm{CL}_{\mathrm{i}-1}$ passes the RREQUEST messages to $\mathrm{CL}$. Once $M_{1}$ receives the message, $M_{i}$ reports the addresses of its slave nodes to $\mathrm{MN}_{\mathrm{i}-1}$ andpasses the RREQUEST to $\mathrm{MN}_{\mathrm{i}+1}$ with co (fuzzy cost).

Step 5: $\mathrm{P}$ is relaiable. The value of $\mu \mathrm{A}(\mathrm{z})=1$. That means $\mathrm{z}$ is completly member of fuzzy set.

Then cost can be calculated by the following formula:

$$
\left.\mathrm{MN}_{\mathrm{i}}^{\mathrm{p}}=\operatorname{Ni}(\mathrm{Mi}(\lambda \mathrm{p})) / \max \left\{\mathrm{N}_{\mathrm{i}}(\lambda \mathrm{p})\right\}_{\mathrm{i}=1}^{\mathrm{n}}\right)
$$

The loop will continue until $\mathrm{P}$ value reaches $\mathrm{L}$ and $\mathrm{i}$ value is reachesC

Step 6: $P$ is unreliable. The value of $\mu \mathrm{A}(\mathrm{z})=0$. that means $\mathrm{z}$ is not fully member of fuzzy set. Then cost can be calculated by the following formula:

$$
\left.\mathrm{MN}_{\mathrm{i}}^{\mathrm{p}}=\operatorname{Ni}(\operatorname{Mi}(\lambda \mathrm{p})) / \max \left\{\mathrm{N}_{\mathrm{i}}(\lambda \mathrm{p})\right\}_{\mathrm{i}=1}^{\mathrm{n}}\right)
$$

The loop will continue until $\mathrm{P}$ value reaches $\mathrm{L}$ and $\mathrm{i}$ value is reachesC.

Step 7: $\mathrm{i}$ value ranges 1 to $\mathrm{N}$ and $\mathrm{P}$ value ranges from 1 to $\mathrm{L}$ (Maximum resources). The total cost can be calculated by the following formula.

$$
\begin{aligned}
& \operatorname{Cos} \mathrm{t}=0 ; \\
& \operatorname{Cos} \mathrm{t}=\mathrm{MN}_{\mathrm{i}}^{\mathrm{p}}+\cos \mathrm{c} ; \\
& \cos \mathrm{t}[\mathrm{i}]=
\end{aligned}
$$

Repeat the loop until reaches the maximum value i.e., $P$ reaches $L$ value and $i$ reaches $C$ value.

Step 8: Then, it sets up multiple disjoint links: $\left\{\mathrm{N}_{\mathrm{i}-}\right.$ $\left.{ }_{1 \mathrm{j}} \rightarrow \mathrm{N}_{1 \mathrm{j}}\right\},\left(\mathrm{N}_{\mathrm{i}-1 \mathrm{j}} \in \mathrm{MN}_{\mathrm{i}-1}, \mathrm{~N}_{1 \mathrm{j}} € \mathrm{MN}_{1}\right)$;

Step 9: It sets up links from the members of $\mathrm{MN}_{\mathrm{i}} \mathrm{So}_{\mathrm{i}}=$ $\left\{\mathrm{N}_{\mathrm{ij}}\right\}$ ( as source nodes) to the members of cluster $\mathrm{CL}_{\mathrm{i}}$ except the $\mathrm{N}_{i j}\left\{\mathrm{MN}_{\mathrm{i}}-\mathrm{N}_{\mathrm{ij}}\right\}$ (as destination nodes), $\left\{\mathrm{MN}_{\mathrm{i}^{-}}\right.$ $\left.\left\{\mathrm{N}_{\mathrm{ij}}-1\right\}\right\}$ as next hop addresses, and chooses the links that satisfies the reliability request ( $\mathrm{co} \geq \mathrm{CO}_{\text {lower }}$ ), the hop of the links is likely more than one;

Step 10: When the master node $\mathrm{MN}_{\mathrm{m}}$ where the destination locate receives the path request message, cluster CLm will set up disjoint multiple links from $\mathrm{So}_{\mathrm{m}}=\left\{\mathrm{MN}_{\mathrm{i}}-\mathrm{Dn}\right\}$ (as source nodes ), Dn $\left(\mathrm{N}_{\mathrm{ij}}\right)=\left\{\mathrm{MN}_{\mathrm{i}-}\left\{\mathrm{N}_{\mathrm{ij}}-1\right\}\right.$ as next hop address, to destination node and choose the links that satisfies the reliability request $\left(\mathrm{co} \geq \mathrm{CO}_{\text {lower }}\right)$ with cost $\mathrm{Co}$.
Step 11: Finally, when all completed paths to destination node have been established, it will choose all maximal disjoint, loop free reliable paths that satisfy above conditions based on hop number and bandwidth.

The above paths just are possible routes, we call them virtual routes.

Reverse Link Labeling:It identifies as real routes which are accompanied by the virtual path and loopfree. It satisfies the QoS requirement for this particular session. Of course while destination Dn generates a one hop broadcast, never fails to send the reverse link labeling message. The following fields which are included in the reverse link labeling message are delineated below.

In Fig. 5 and 6 the reverse-link labeling phase, Dn sets SL as its IP address, $\mathrm{HO}$ and $\mathrm{AD}$ as 0 while other fields are the same with those in the route request message. Every node that receives the reverse labeling message checks whether it meets the following conditions in order to broadcast the packet promptly:

- Ho value Increasing by 1

- $\quad$ adding its delay to AD

- $\quad$ recording $\mathrm{SL}, \mathrm{Ho}, \mathrm{AD}$ and $\mathrm{co}_{\mathrm{i}}$ into its routing table

\begin{tabular}{|l|}
\hline Source address (So) \\
\hline Destination address (Dn) \\
\hline Session ID \\
\hline $\mathrm{P}_{\text {lower }}$ \\
\hline QoS request \\
\hline Virtual Path (VP) \\
\hline
\end{tabular}

Fig.5: RREQUEST

\begin{tabular}{|l|}
\hline Source Address (So) \\
\hline Labeling Source Address (S L ) \\
\hline Session ID \\
\hline QoS Requirements \\
\hline Virtual Path (VP) \\
\hline Hop (Ho) \\
\hline Accumulated Delay (AD ) \\
\hline Fuzzy cost (co $\left.{ }_{i}\right)$ \\
\hline
\end{tabular}

Fig.6: RREPLY 
Thus, more than one route will be discovered between So and Dn that comprise of links labeled by session ID.

Route Strategy and Traffic Distribute: When source node receives the RREPLY messages, it sets up multiple paths from source node to destination node. However these paths are real paths. According to the Hop number (ho), Accumulated Delay (AD), Bandwidth (bw) and fuzzy Cost $\mathrm{co}_{\mathrm{i}}$ included in the paths messages received by source. In these paths are classified such as optimal path and shortest path.

Dynamic Route Rectification and Maintanance:If a slave node node fails to receive three messages continuously from its master node and to locate a new master node, it becomes itself a master node. And the node will delete the route which is broken from the routing table. It has to select the new route that meets the QoS requirement.

\section{MATERIALS AND METHODS}

This model is implemented with NS2 simulator with radio probagation rate $2 \mathrm{mbps}$. In our proposed protocol 100 mobile nodes are placed randamly in a region of $1500 \mathrm{~m} \times 500 \mathrm{~m}$ with a maximum and minimum speed from $5 \mathrm{~ms}$ to $10 \mathrm{~ms}$. The transmission range is 250 meters for all nodes. The excution time is 900 seconds for each simulation.

\section{RESULTS}

Figure 7 shows the result about number of routing discovery phases against the mobility. The frequency of routing invention and identification for the multipath routing FCMR and CBMR is almost the same and is not more than that for the unipath routing
approach.FCECR plays an important role in minimizing the cost in route discovery and deleting broken routes.

Figure 8 it is states that there are results about average end-to-end delay, which causes for the queue delay in every host and the propagation delay from the source to the destination. So multipath routing FCECR results in reduction of the queue delay by reason of which traffic is distributed through various tracks. If not, it naturally increases the propagation delay because a few data packets are forwarded along the sub-optimal paths.

From Figure 9, is not that the unipath routing have higher average point-to-point delay in compensation with multipath routing and the average point-to-point delay of FCECR is higher than that of CBMR and FCMR. From the demonstration it is understood that the multipath routing control distributes the traffic and improve the end-to-end delay and the improvement in limited below pause time of 300 seconds. The network topology changes more frequently at smaller pause time and hence the average end-to-end delay for the both multipath routing and unipath routing decreases. Thus queuing delay of the data packets in the source node increases which leads the increase of the average endto-end delay.

\section{DISCUSSION}

In this study of our article, it is observed and determined that fuzzy cost enabled cluster based routing algorithm takes much concentration on selecting appropriate path on cluster based multipath routing at fuzzy cost. It is an ultimate conclusion that the method helps us to reduce the cost and route discovery overhead expenses and it is advisable that fuzzy cost enabled cluster based routing algorithm for mobile ad hoc networks that can be effectively utilized at large level.

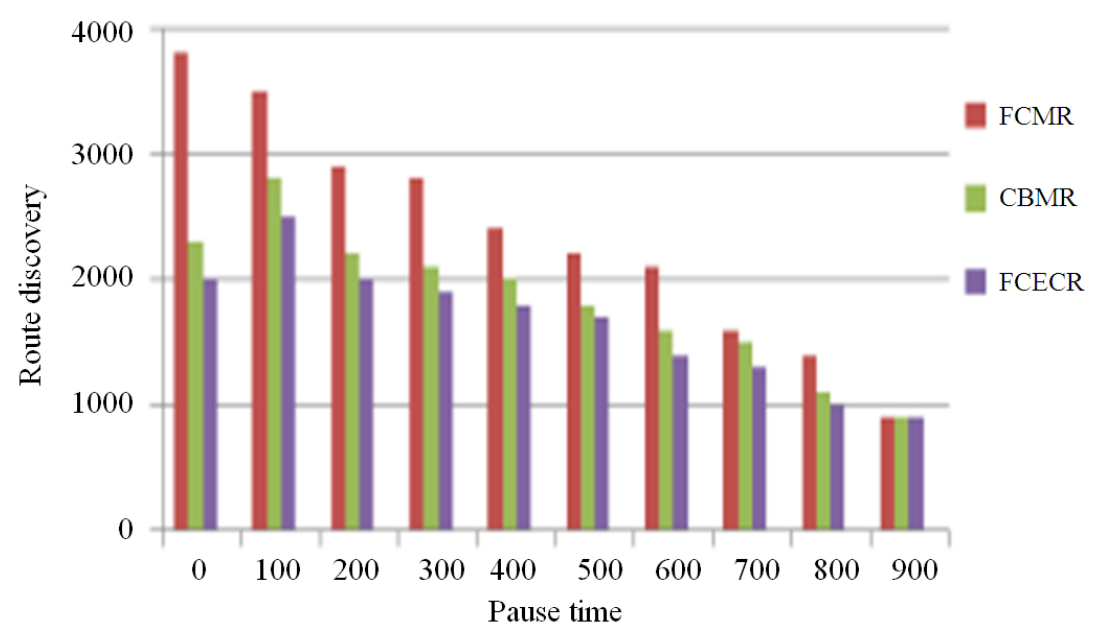

Fig. 7: Route discovery 


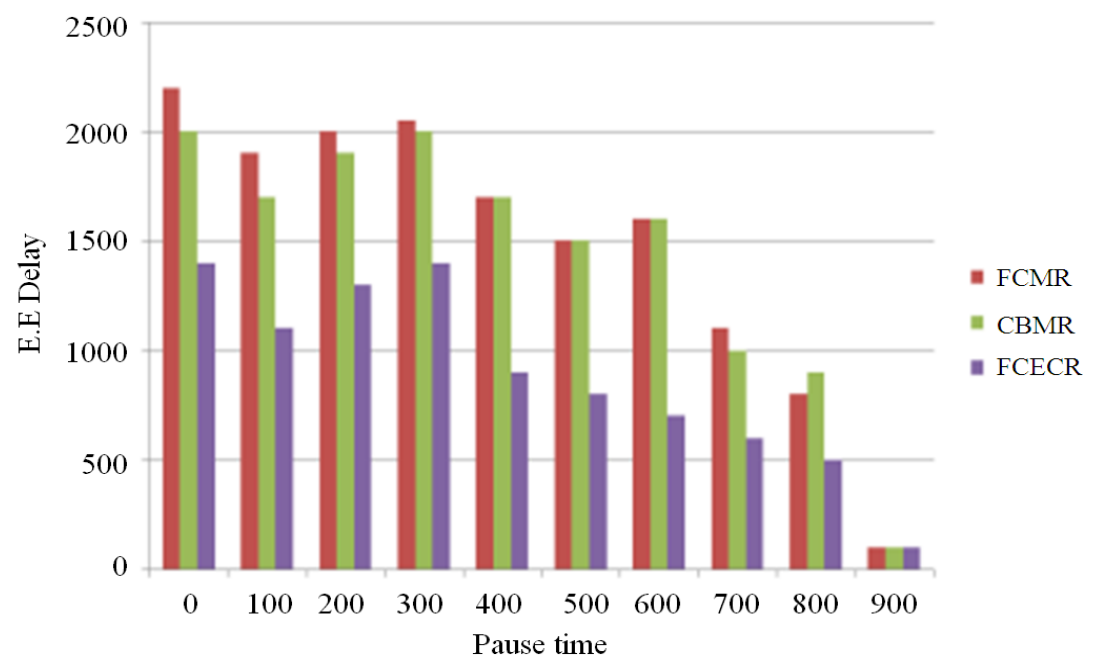

Fig. 8: End-End delay

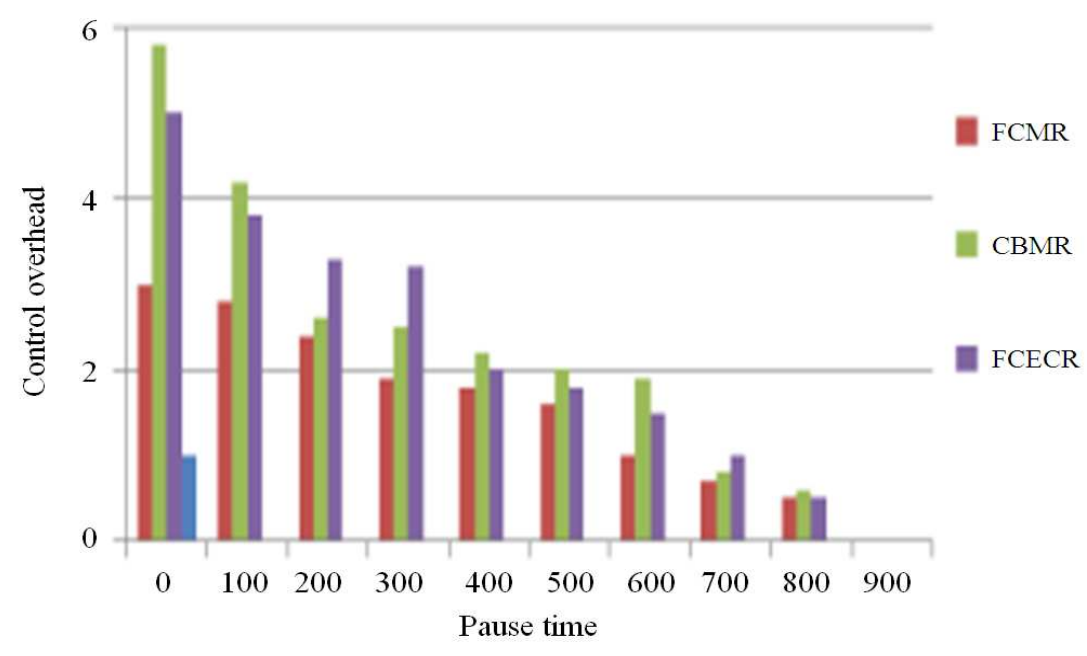

Fig. 9: Control overhead

\section{CONCLUSION}

On the analysis, observation and study of my article, it is recommended that proposed method reduces the cost and route discovery overhead. Benefits are supportable for an ideal routing approach for MANET. It is possible to evaluate the performance of the proposed algorithm on the basis of parallel architectures in future.

\section{REFERENCES}

Raju, A.N. and S. Rmachandram, 2008. Fuzzy Cost Based Multipath Routing For Mobile Ad-Hoc Networks. Institute of Technology and Science Osmania University.
Perkins, C.E. and P. Bhagwat, 1994. Highly dynamic Destination-Sequenced Distance-Vector routing (DSDV) for mobile computers. Proceedings of the Conference on Communications Architectures, Protocols And Applications, (CAPA' 94), ACM Press, USA, pp: 234-244. DOI: 10.1145/190314.190336

Mohamed, H. and M.H. Lee, M. Sarahintu, S. Salleh and B. Sanugi, 2008. The use of Taguchi method to determine factors affecting the performance of destination sequence distance vector routing protocol in mobile ad hoc networks. J. Math. Stat., 4:

194- 
Hsi, O.C. and A. Abdullah, 2009. Performance evaluation of stable weight-based on demand routing protocol for mobile ad hoc network. J. Comput. Sci., 5: 732-737. DOI: $10.3844 /$ jcssp.2009.732.737
Park, S. and S.M. Yoo, 2010. Routing table maintenance in mobile ad hoc networks. Proceedings of the 12th International Conference on Advanced Communication Technology, Feb. 710, IEEE Xplore Press, pp: 1321-1325. 